\title{
Assessment of discharge treatment prescribed to women admitted to hospital for hyperemesis gravidarum
}

\author{
L. Fiaschi*a, G. Housley ${ }^{b}$, C. Nelson-Piercyc, J. Gibson a, Ayokunnu Raji ${ }^{a}$, S. Deb ${ }^{\text {d }}$, L. J. Tata ${ }^{a}$
}

*Corresponding author: linda.fiaschi@nottingham.ac.uk. t: +44 (0) 1158231250

ORCiD: 0000-0002-3780-5895

a Division of Epidemiology \& Public Health, University of Nottingham, Clinical Sciences Building, City Hospital, NG5 1PB Nottingham, UK

${ }^{b}$ Nottingham University Hospitals and East Midlands Academic Health Science Network, Nottingham, England

' Women's Health Academic Centre, Guy's \& St Thomas' Foundation Trust, St Thomas' Hospital, SE1 7EH London, United Kingdom

d Nottingham University Hospital Department of Obstetrics and Gynaecology Queen's Medical Centre Derby Road, NG7 2UH Nottingham.

Disclosure

C.N.P. reports personal fees from Sanofi Aventis, Warner Chilcott, Leo Pharma, UCB and Falk, outside the submitted work, and from Alliance Pharma as consultancy fees within the submitted work. She is also one of the co-developers of the RCOG Green Top Guideline on HG. All other authors did not report any potential conflicts of interest. 


\section{Abstract}

Aims: Prescribing drug treatment for the management of hyperemesis gravidarum (HG), the most severe form of nausea and vomiting in pregnancy, remains controversial. Since most manufacturers do not recommend prescribing antiemetics during pregnancy, little is known regarding which treatments are most prevalent among pregnant patients. Here we report for the first time, evidence of actual treatments prescribed in English hospitals.

Methods: A retrospective pregnancy cohort was constructed using anonymised electronic records in the Nottingham University Hospitals Trust system for all women who delivered between January 2010 and February 2015. For women admitted to hospital for HG, medications prescribed on discharge were described and variation by maternal characteristics was assessed. Compliance with local and national HG treatment guidelines was evaluated.

Results: Of 33,567 pregnancies (among 30,439 women), the prevalence of HG was 1.7\%. Among $530 \mathrm{HG}$ admissions with records of discharge drugs, Cyclizine was the most frequently prescribed (almost $73 \%$ of admissions). Prochlorperazine and metoclopramide were prescribed mainly in combination with other drugs, however, ondansetron was more common than metoclopramide at discharge from first and subsequent admissions. Steroids were only prescribed following readmissions. Thiamine was most frequently prescribed following readmission while high dose of folic acid was prescribed equally after first or subsequent admissions. Prescribing showed little variation by maternal age, ethnicity, weight, socioeconomic deprivation, or comorbidities.

Conclusion: Evidence that management of HG in terms of discharge medications mainly followed local and national recommendations provides reassurance within the health professional community. Wider documentation of drugs prescribed to women with HG is required to enable full assessment of whether optimal drug management is being achieved.

\section{What's known?}

- Local and national guidelines on hyperemesis gravidarum (HG) management recommend a stepwise approach to treatment medications based on the level of HG severity

- Manufacturers worldwide remain cautious and do not recommend prescribing most antiemetics during pregnancy

What's new?

- Drug management of HG in a large English hospital trust reflects national guidelines

- Discharge prescriptions indicated that stepwise use of treatments based on HG severity was being used universally; use of first, second and subsequent-line treatments showed little variation by maternal characteristics such as age, ethnicity or deprivation 


\section{Introduction}

Hyperemesis Gravidarum (HG), the most severe form of nausea and vomiting in pregnancy (NVP), is characterised by persistent vomiting resulting in fluid and electrolyte derangement, weight loss and nutritional deficiencies severe enough to require medical attention. [1] The reported occurrence of HG, varies between $0.3 \%$ to $2 \%$ of pregnancies worldwide. [2] HG is the commonest cause of hospital admission in pregnancy, after preterm labour, in the United Kingdom (UK) and the United States[3,4] and the costs associated with the burden of managing HG are significant worldwide. [5-7]

A wide range of therapeutic agents are used in the management of HG internationally. [7-9] In the UK, the 2016 Royal College of Obstetricians and Gynaecologists (RCOG) Green Top Guidelines [10] recommend a stepwise prescribing system where use of antihistamines and phenothiazines are defined as first line treatment; dopamine and serotonin antagonists as second line treatments, followed by steroids as third line treatment; the use of pyridoxine is discouraged. Although several systematic reviews [11-13] provide evidence for the safety of antihistamine and dopamine antagonists used in pregnancy, manufacturers worldwide remain cautious and do not recommend their use antenatally, leaving prescribing to the discretion of the health professional when they consider the potential benefits outweigh the risks. In light of this, it is not yet known which drug treatments are most commonly used to treat $\mathrm{HG}$, or whether and how current clinical guidelines are followed by health professionals. An understanding of the common clinical practice adopted by health professionals could help increase confidence in doctors' prescribing, highlight potential weaknesses of the actual management and therefore help improve the service offered to women affected by HG.

English Maternity Hospital Episode Statistics provide important information on HG admissions for almost all pregnancies in England[14,2], however, they currently have no information on medications prescribed. To determine which drugs are most commonly prescribed at discharge for the management of HG in secondary care, we used anonymised clinical data from women admitted to the Nottingham University Hospitals (NUH) National Health Service Trust. We evaluated whether prescribing reflected national or local guidelines and whether prescribing differed based on women's characteristics, such as age and ethnicity.

\section{Methods}

\section{Study population}

This study used anonymised clinical data from NUH, which is one of the largest National Health Service (NHS) Hospital Trusts England, accounting for roughly 10,000 births a year. [15] An NHS hospital trust is a secondary care organisation, typically consisting of 1-3 collectively managed hospitals that cover a geographical catchment area for the local population. As part of England's universal public health care system (the NHS), all medical care is free at the point of hospital admission and prescription medications are free of charge during pregnancy. All pregnant women booked for antenatal care who delivered in an NUH facility between January 2010 and February 2015 were included. Data were captured, combined across different hospital databases and anonymised for research purposes. Pregnancies without information on gestational week at delivery (35\%) due to under-recording, were excluded. This did not affect the results of our analysis as the distribution of maternal characteristics for women with known and unknown gestational age were similar (results not shown). Women 
admitted to hospital with a primary diagnosis of HG were identified using International Classification of Diseases version 10 (ICD-10) [16] codes O210 and O211. Data and ethical approval were obtained through the East Midlands Academic Health Science Network (EMAHSN). [17]

\section{HG treatment medications}

Prescriptions were extracted from NUH's electronic discharge summary system, developed in-house to facilitate recording of drugs prescribed for the patient to take home, electronically issued to their general practitioner. We classified medications prescribed at hospital discharge for HG admissions according to the NUH HG management guidelines[18] as follows: antihistamines, phenothiazines, dopamine antagonists, serotonin antagonists, corticosteroids and vitamins. Guidelines for HG management, both nationally[10] and at NUH[18] follow a stepwise approach based on the level of HG severity, response to treatment and current evidence of drug safety. The Green Top Guidelines[10] present recognised methods and techniques for clinical practice based on published evidence and are considered the national gold standard recommendations for health professionals in the UK. Drug classes typically prescribed in the UK[10] for HG management and sequential lines of action are shown in Table 1.

We assessed the following maternal factors related to HG prescribing based on previous evidence of HG risk factors [2,19]: maternal age, ethnicity, body mass index (BMI), smoking status, number of previous pregnancies, birth plurality, socioeconomic deprivation as measured by the Index of Multiple Deprivation (IMD 2010) [20] and the following maternal comorbidities: diabetes (including type1, 2 and gestational), asthma and mental health problems. Women were defined with mental health problems if they had or have previously had mental health issues, such as postnatal depression, stress, eating disorders, or self-harm. Maternal characteristics were recorded during the first appointment with a midwife which is usually around week 10 of gestation.

\section{Analysis of drug prescribing}

We plotted the occurrence of HG admissions by gestational week to assess that the distribution followed the recognised presentation of $\mathrm{HG}$ across pregnancy. For $\mathrm{HG}$ admissions with recorded drug prescriptions at discharge, we described the prescribing prevalence for each of the 6 drug classes at first and subsequent HG admissions. Prescribing prevalence at first admission was assessed separately for women who only had one admission and women who went on to be readmitted. We then described the prescribing prevalence of individual drugs by admission according to whether they were first line, second line, third line or fourth line treatment in the NUH clinical guidelines. [18] We included separate prevalence figures to show whether drug classes (or individual drugs) were prescribed alone or in combination. To assess whether stepwise prescribing according to the guidelines was being used universally for all women admitted with HG, we compared proportions prescribed each line of treatment across each maternal characteristic using chi-squared or Fisher's exact tests. 
Results

There were 51,613 pregnancies in the NUH pregnancy cohort between January 2010 and February 2015, of which $33,567(65 \%)$ had a usable record of gestation. The prevalence of being admitted for HG was $1.4 \%$ in the overall population $(740 / 51,613)$ and $1.7 \%$ among pregnancies with gestational week at delivery recorded $(571 / 33,567)$. For those with gestational records, HG admissions peaked at 8 weeks of gestation and the median length of hospital stay was 2 days with an interquartile range of 1-3 (Fig 1).

\section{Drug prescribing on discharge}

There were 1,037 admissions with a primary diagnosis of HG, of which 530 (51\%) had records for prescribed drugs at discharge. Antihistamines, prescribed either alone or in combination were the most common drug class prescribed at discharge from first HG admissions, followed by serotonin antagonists (Table 2). Antihistamine prescriptions were less frequent for subsequent admissions but they were still the most commonly prescribed drug class alone. Dopamine and Serotonin antagonists were most commonly prescribed in combination at discharge from subsequent admissions. Prescribing prevalence of phenothiazines was similar following first and subsequent admissions. Vitamins were prescribed on discharge more often in subsequent admissions, whilst steroids were used exclusively at subsequent admissions. First and subsequent admissions differed considerably for each drug class prescribed at discharge, except for phenothiazine. All drugs were more commonly used in combination than alone. Compared with women who were only admitted once, women with subsequent admissions were more likely to have been discharged after their first admission with drugs other than dopamine antagonists in combination.

In the context of the NUH guideline for HG management, Table 3 shows the frequency of discharge prescriptions according to each line of treatment. While promethazine was very rarely prescribed, cyclizine was generally the most common first line drug, prescribed alone or in combination. Most first admissions had a discharge prescription for cyclizine (79\% of those among women admitted only once and $83 \%$ of those for women with multiple admissions) as did subsequent admissions (9\% prescribed alone and 53\% in combination) (Table 3). In terms of second line treatments, prochlorperazine was prescribed less often than metoclopramide at discharge. Use of prochlorperazine was prescribed similarly at first and subsequent admissions, whereas discharge prescribing of metoclopramide was more common in subsequent admissions. Ondansetron, which is recommended as third line treatment in NUH guidance was in fact more common than metoclopramide for first admissions both alone and in combination with other drugs. It was also prescribed more often for discharge from subsequent admissions compared with first admissions.

The only prescribed steroid was prednisolone and, in line with NUH guidelines, was only prescribed at discharge from readmission. Thiamine was more frequently prescribed following readmissions compared with first admissions, in line with NUH guidelines, while high dose of folic acid was prescribed similarly following first or subsequent admissions. There were no prescriptions for chlorpromazine, hydrocortisone nor methylprednisolone, however, these drugs are usually given intravenously and therefore not prescribed at discharge. For first admissions, the prescribing prevalence of cyclizine, prochlorperazine and ondansetron were considerably higher, particularly in combination, among women who went on to have subsequent admissions, compared with women admitted only once. The most commonly prescribed drug combinations were cyclizine 
with ondansetron, metoclopramide with cyclizine and metoclopramide with ondansetron, accounting for $40 \%$, $19 \%$ and $19 \%$ of all admissions, respectively.

Discharge prescriptions indicated that stepwise use of treatments based on HG severity was being used universally (Table 4). First line therapy was prescribed most often, however third line therapy (ondansetron), was prescribed more frequently than second line therapy. Use of first line, second line and subsequent line treatments showed little variation by maternal characteristics as shown in Table 4 where the percentages are calculated over the total for each maternal characteristic value. Use of first line therapy was slightly more common in women with recorded mental health problems. Younger women were slightly less likely to receive discharge prescriptions for second line treatments, although there were no statistically significant differences for any other lines of treatment according to age. Lower BMI and non-smoking were associated with slightly higher use of third line therapy. Fourth line treatment did show variation according to certain maternal characteristics, such as deprivation and smoking status, however it was very rarely prescribed at discharge (only 21 admissions) so statistical power was low for assessing differences. Thiamine was more commonly prescribed in women with Asian or Other ethnicity recorded, and less commonly prescribed in women with previous mental health problems.

\section{Discussion}

\section{Main findings}

At discharge from HG admission, drugs were most often prescribed in combination rather than alone. The drugs most frequently prescribed, in order of occurrence, were cyclyzine (first line treatment), ondansetron (third line treatment), metoclopramide and prochlorperazine (second line treatments). In line with local hospital guidelines prednisolone (fourth line treatment) was prescribed only at discharge from readmission. While thiamine was most frequently prescribed following readmission, high dose of folic acid was equally used at first and subsequent admissions. Prescribing patterns were generally very similar across all women, regardless of differences in maternal characteristics. Only prescribing of third and fourth line treatments, for the most severe presentations of HG, showed some variation by women's characteristics; these were more likely prescribed to women with asthma, those who were underweight, or non-smokers.

\section{Strengths and limitations}

This is the first study to assess what drugs are prescribed at discharge from hospital in England to women affected by HG. We used routinely recorded electronic patient records from one of the largest local hospital trusts in England, which included over 30,000 pregnancies extracted via the East Midlands Academic Health Services Network. The hospital trust serves the whole local population and therefore, unless women are travelling away from home, any admission for HG should be captured in our study, thus minimizing selection bias of the local Nottinghamshire population. Nottinghamshire, however, has slightly higher socioeconomic deprivation compared with the national average so this population is not thoroughly representative of pregnancies across the country. The available National Maternity Hospital Episode Statistics (HES), however, currently lacks any information on drug prescriptions so it is not possible to conduct a national study. 
Inpatient prescriptions are recorded in the patient's paper records and may be recorded in the pharmacy dispensary system but if the item is obtained from ward stock it is not explicitly linked to a patient's record electronically. In the absence of a full Electronic Prescribing and Medicines Administration (EPMA) system and without manual checks of paper records, the use of discharge prescriptions was considered the best option for assessing drug information which was uniquely linked to a patient. Clinical practice indicates that discharge medications are the same as those that would have been used during hospital admissions in their parenteral form. Drugs prescribed at discharge would capture the final successful medication that allowed the patient to be discharged from hospital, reflecting the adequate line of treatment for the severity of their HG. This could have been the first treatment attempt by health professionals or the final one, if previous lines of action were inadequate. Some drugs such as steroids are mainly given intravenously and therefore more likely to be prescribed during hospital stay rather than at discharge. Of all admissions, $49 \%$ did not have information on prescribed drugs at discharge and therefore were not included in this analysis. In UK clinical practice, women with HG are rarely discharged from hospital without antiemetics prescriptions (expert opinion from CNP and S; SD manages NUH HG clinic admissions) therefore this missing information is attributable to suboptimal recording. A likely common reason for the lack of recording is that when women are admitted for fewer than 24 hours and discharged with the same medications as those they had at admission, the medication is not routinely re-recorded in the electronic discharge letter. We acknowledge that this exclusion could impact significantly on the results in terms of underestimation of cases who were on first or second line treatment previously prescribed by their general practitioner, or by the hospital if they had been admitted previously (one third of the admissions without drugs information). Moreover, the electronic discharge summary system was not universally used before 2012 therefore some prescriptions may have not been included.

One third of pregnancies did not have information on gestational age, similar to national Maternity HES data[2], and although we have no reason to think the information was not missing at random, multiple imputation of missing values could not be applied as the missing information for many variables affected the same group of women.

\section{Comparisons with previous studies}

We found a prevalence of $1.7 \%$ for being admitted to hospital for HG during pregnancy (1.4\% among pregnancies overall including those with missing gestational age) which was similar to figures from other international studies[21-23] ranging between 0.3 and 1.5\%, and our previous study using national data[24]. We found a median length of hospital stay of 2 days for any admission, within the 1.8 to 6 day range reported in the literature[25-27]. Length of hospital stay is linked to the effectiveness of the specific treatment offered during the current hospital stay, the severity of the HG episode as well as different local policies and guidelines, as treatment for HG is not internationally standardized. The NUH Trust has recently implemented a day-case unit for HG management which has been elsewhere[28] shown to decrease the length of stay.

While a recent systematic review[29] assessed the effectiveness of treatments for NVP and HG, no evidence has yet been published on the actual drugs prescribed in English hospitals. We found that antihistamines along with serotonin antagonists were the most commonly prescribed discharge treatments for patients admitted with $\mathrm{HG}$ regardless of whether it was a first or a subsequent admission. This is in general agreement with HG medication use reported by 765 women from 26 countries [8] who filled out an online survey on the Hyperemesis Education 
and Research Foundation website, although this showed that women from the UK were more likely to be treated with phenothiazines rather than antihistamines. The use of self-reports, however, can affect the accuracy and generalisability of the results depending on who participated in the survey. NUH guidelines recommend ondansetron as third line treatment[18] however, the actual management shows it was more commonly prescribed than metoclopramide (second line treatment) at discharge for first and subsequent admissions. This could be due to women having been previously treated in an out-patient setting with second line drugs in an attempt to avoid hospital admission or it could suggest a shift in ondansetron usage towards a second line treatment, in agreement with the national RCOG guidelines published later in 2016. [10]

When we assessed prescribing at first admission, women who went on to have readmissions were more likely to be prescribed second and third line treatments and to receive drugs in combination, compared with women who had no further admissions. Rather than potential sub-optimal treatment, this could reflect a higher level of $\mathrm{HG}$ severity in women with subsequent readmission, supporting the hypothesis that for severe cases of $\mathrm{HG}$, readmissions cannot always be prevented.

Only a few studies have so far described maternal characteristics of women by antiemetic use. In general we found modest variation in prescribing according to women's characteristics, however, asthmatic women were more likely to be prescribed corticosteroid; it is possible that some of this use was related to asthma which is slightly more common in women presenting with HG. Thiamine was more common in women with black or Asian ethnicity, who are groups with particularly high risks of HG. [2] We found that older women were more likely to be prescribed phenothiazine and dopamine antagonists while women who were underweight or nonsmokers were more likely to be prescribed serotonin antagonists, in contrast with two previous North American studies from 2013[30] and 2014[31], which both reported a higher usage of antihistamines among older women. An Australian study reported that serotonin antagonists were more likely prescribed to non-smoking women in agreement with our results. [23] The same study also found they were used more for women in higher socioeconomic groups and Caucasian women, in contrast to our study. [32] To the best of our knowledge, no study to date has assessed whether hospital prescribing for HG is compliant with national or local guidelines in England.

\section{Conclusions}

The use of discharge medications for the clinical management of HG in a large English hospital trust showed that first line treatment was the most commonly prescribed. However, there was more prescribing of third line treatment (ondansetron) compared to second line (metoclopramide), showing a closer compliance with national rather than local guidelines. While there was a reticence towards promethazine and prochlorperazine treatment, steroids were confirmed to be rarely prescribed, being limited mainly to women who were readmitted.

This evidence from routine professional practice in antiemetic prescribing during gestation helps increase knowledge and confidence in implementing and delivering optimal management to women affected by HG, although we still need further information on drug prescribing for HG in primary and secondary care settings. It would be particularly useful to collect fetal and neonatal outcomes following prescriptions of different drug classes. Urgent calls are needed to fully develop and optimize comprehensive electronic prescribing systems 
within secondary care settings at national level, a key data component which is currently missing for studying drug safety in pregnancy.

\section{Authors' contribution}

LJ Tata: Protocol and project development, Methodology, Manuscript editing, Interpretation of the results and critical review.

L Fiaschi: Protocol and project development, Data management, Data analysis, Methodology, Manuscript writing, Interpretation of the results and critical review.

C Nelson-Piercy: Protocol and project development, Methodology, Manuscript editing, Interpretation of the results and critical review.

G Housley: Protocol and project development, Data collection and management, Manuscript editing, critical review.

J Gibson: Methodology, Interpretation of the results and critical review

A Raji: Methodology, Data management, Manuscript editing, critical review.

S Deb: Methodology, Manuscript editing, Interpretation of the results and critical review

\section{Acknowledgments}

The work was funded by Nottingham Hospitals Charity and the Department of Research and Innovation. The funders had no role in study design, data collection and analysis, decision to publish, or preparation of the manuscript. Grant number RB48TW. Data collection and ethical approval was received from the East Midlands Academic Health Sciences Network.

\section{References}

[1] Jarvis S, Nelson-Piercy C. Management of nausea and vomiting in pregnancy. BMJ 2011;342:d3606d3606. doi:10.1136/bmj.d3606.

[2] Fiaschi L, Nelson-Piercy C, Tata LJ. Hospital admission for hyperemesis gravidarum: a nationwide study of occurrence, reoccurrence and risk factors among 8.2 million pregnancies. Hum Reprod 2016;31:167584. doi:10.1093/humrep/dew128.

[3] Gazmararian JA, Petersen R, Jamieson DJ, Schild L, Adams MM, Deshpande AD, et al. Hospitalizations During Pregnancy Among Managed Care Enrollees. Obstet Gynecol 2002;100:94-100.

[4] Dean C. Helping women prepare for hyperemesis gravidarum. Br J Midwifery 2014;22:847-52. doi:10.12968/bjom.2014.22.12.847.

[5] Bailit JL. Hyperemesis gravidarium: Epidemiologic findings from a large cohort. Am J Obstet Gynecol 2005;193:811-4. doi:10.1016/j.ajog.2005.02.132.

[6] Trovik J, Vikanes $\AA$. Hyperemesis Gravidarum is associated with substantial economic burden in addition to severe physical and psychological suffering. Isr J Health Policy Res 2016;5:43. doi:10.1186/s13584016-0099-y.

[7] O’Donnell A, McParlin C, Robson SC, Beyer F, Moloney E, Bryant A, et al. Treatments for hyperemesis gravidarum and nausea and vomiting in pregnancy: a systematic review and economic assessment. Health Technol Assess Winch Engl 2016;20:1-268. doi:10.3310/hta20740.

[8] Goodwin T, Poursharif B, Korst L, MacGibbon K, Romero R, Fejzo M. Secular Trends in the Treatment of Hyperemesis Gravidarum. Am J Perinatol 2008;25:141-7. doi:10.1055/s-2008-1040344.

[9] Markl GE, Strunz-Lehner C, Egen-Lappe V, Hasford J. Prescribing patterns of anti-emetic drugs during pregnancy in Germany. Arch Gynecol Obstet 2007;275:461-7. doi:10.1007/s00404-006-0286-0. 
[10] RCOG. The Management of Nausea and Vomiting of Pregnancy and Hyperemesis Gravidarum (Greentop Guideline No. 69). R Coll Obstet Gynaecol 2016. https://www.rcog.org.uk/en/guidelines-researchservices/guidelines/gtg69/ (accessed May 17, 2017).

[11] Matthews A, Dowswell T, Haas DM, Doyle M, O’Mathúna DP. Interventions for nausea and vomiting in early pregnancy. Cochrane Database Syst Rev 2010:CD007575. doi:10.1002/14651858.CD007575.pub2.

[12] Magee LA, Mazzotta P, Koren G. Evidence-based view of safety and effectiveness of pharmacologic therapy for nausea and vomiting of pregnancy (NVP). Am J Obstet Gynecol 2002;186:S256-61. doi:10.1067/mob.2002.122596.

[13] Mazzotta P, Magee DLA. A Risk-Benefit Assessment of Pharmacological and Nonpharmacological Treatments for Nausea and Vomiting of Pregnancy. Drugs 2000;59:781-800. doi:10.2165/00003495200059040-00005.

[14] Health and Social Care Information Centre. Hospital Episode Statistics 2012. http://www.hscic.gov.uk/hes (accessed May 24, 2016).

[15] NHS Digital UK. Hospital Maternity Activity, 2015-16 2016. http://www.content.digital.nhs.uk/catalogue/PUB22384 (accessed June 27, 2017).

[16] World Health Organization. International Classification of Diseases, Version 102010. http://apps.who.int/classifications/icd10/browse/2010/en.

[17] Home page. EMAHSN n.d. http://emahsn.org.uk/ (accessed March 2, 2017).

[18] Deb S, Shorter K. Guideline on management of hyperemesis gravidarum - Nottingham University Hospitals NHS Trust n.d. https://www.nuh.nhs.uk/handlers/downloads.ashx?id=61187 (accessed February 28, 2017).

[19] Roseboom TJ, Ravelli ACJ, van der Post JA, Painter RC. Maternal characteristics largely explain poor pregnancy outcome after hyperemesis gravidarum. Eur J Obstet Gynecol Reprod Biol 2011;156:56-9. doi:10.1016/j.ejogrb.2011.01.010.

[20] Department for Communities and Local Government and The Rt Hon Eric Pickles. English indices of deprivation - Publications 2010. https://www.gov.uk/government/statistics/english-indices-of-deprivation2010 (accessed May 28, 2015).

[21] Verberg MFG, Gillott DJ, Al-Fardan N, Grudzinskas JG. Hyperemesis gravidarum, a literature review. Hum Reprod Update 2005;11:527-39. doi:10.1093/humupd/dmi021.

[22] Vikanes A, Grjibovski AM, Vangen S and Magnus P. Variations in prevalence of hyperemesis gravidarum by country of birth: A study of 900,074 pregnancies in Norway, 1967-2005. Scand J Public Health Mar2008;36:7.

[23] Bolin M, Åkerud H, Cnattingius S, Stephansson O, Wikström A. Hyperemesis gravidarum and risks of placental dysfunction disorders: a population-based cohort study. BJOG Int J Obstet Gynaecol 2013;120:541-7. doi:10.1111/1471-0528.12132.

[24] Fiaschi L, Nelson-Piercy C, Tata LJ. Hospital admission for hyperemesis gravidarum: a nationwide study of occurrence, reoccurrence and risk factors among 8.2 million pregnancies. Hum Reprod 2016:dew128.

[25] Ditto A, Morgante G, la Marca A, De Leo V. Evaluation of treatment of hyperemesis gravidarum using parenteral fluid with or without diazepam. A randomized study. Gynecol Obstet Invest 1999;48:232-6. doi:10189.

[26] Lacasse A, Lagoutte A, Ferreira E, Bérard A. Metoclopramide and diphenhydramine in the treatment of hyperemesis gravidarum: effectiveness and predictors of rehospitalisation. Eur J Obstet Gynecol Reprod Biol 2009;143:43-9. doi:10.1016/j.ejogrb.2008.11.007.

[27] Tsang IS, Katz VL, Wells SD. Maternal and fetal outcomes in hyperemesis gravidarum. Int J Gynaecol Obstet Off Organ Int Fed Gynaecol Obstet 1996;55:231-5.

[28] McCarthy FP, Murphy A, Khashan AS, McElroy B, Spillane N, Marchocki Z, et al. Day Care Compared With Inpatient Management of Nausea and Vomiting of Pregnancy: A Randomized Controlled Trial. Obstet Gynecol 2014;124:743-8. doi:10.1097/AOG.0000000000000449.

[29] McParlin C, O’Donnell A, Robson SC, Beyer F, Moloney E, Bryant A, et al. Treatments for Hyperemesis Gravidarum and Nausea and Vomiting in Pregnancy: A Systematic Review. JAMA 2016;316:1392-401. doi:10.1001/jama.2016.14337.

[30] Li Q, Mitchell AA, Werler MM, Yau W-P, Hernández-Díaz S. Antihistamine Use in Early Pregnancy and Risk of Birth Defects. J Allergy Clin Immunol Pract 2013;1:666-74.e1. doi:10.1016/j.jaip.2013.07.008.

[31] Aldridge TD, Hartmann KE, Michels KA, Velez Edwards DR. First-trimester antihistamine exposure and risk of spontaneous abortion or preterm birth. Pharmacoepidemiol Drug Saf 2014;23:1043-50. doi:10.1002/pds.3637.

[32] Colvin L, Gill AW, Slack-Smith L, Stanley FJ, Bower C. Off-label use of ondansetron in pregnancy in Western Australia. BioMed Res Int 2013;2013:909860. doi:10.1155/2013/909860. 
Figure legends

Fig 1 Hospital admissions by gestational week

Table legends

Table 1. Drugs typically prescribed for the management of hyperemesis gravidarum

Table 2. Medication classes prescribed at discharge following 530 hyperemesis gravidarum admissions with recorded drug prescriptions

Table 3. First, second and third line treatment* prescribed at discharge following 530 hyperemesis gravidarum admissions with recorded drug prescriptions

Table 4. Maternal characteristics by NUH line of treatment prescribed at discharge for 530 hyperemesis gravidarum admissions 